\title{
A BAYESIAN VIRTUAL METROLOGY FOR QUALITY INSPECTION OF MOBILE REPEATER SYSTEMS
}

\author{
Sung Do Kim, Jong So Kim, Byeong Min Mun, Suk Joo Bae \\ Department of Industrial Engineering Hanyang University, Seoul, Republic of Korea
}

Corresponding author:

Suk Joo Bae

Department of Industrial Engineering

Hanyang University

17 Haengdang-dong Seongdong-gu, Seoul, Republic of Korea

phone: (+82) 2220-0473

e-mail:sjbae@hanyang.ac.kr

Received: 1 July 2016 Accepted: 28 July 2016

\begin{abstract}
The technology of wideband code division multiple access (WCDMA) has been applied to band selective interference cancellation system (ICS) repeaters. To inspect the telecommunication quality of the systems, quality engineers must check the shape of the signals at the corresponding frequency band of the repeaters. However, measuring the signal quality is a repetitive manual task which requires much inspection time and high costs. In the case of small-sized samples, such as the example of an ICS repeater system, Bayesian approaches have been employed to improve the estimation accuracy by incorporating prior information on the parameters of the model in consideration. This research proposes a virtual method of quality inspection for products using a correlation structure of measurement data, mainly in a Bayesian regression framework. The Bayesian regression model derives prior information from historical measurement data to predict measurements of other frequency bandwidths by exploiting the correlation structure of each measurement data. Empirical results show the potential for reducing inspection costs and time by predicting the values of adjoining frequency bandwidths through measured data of a frequency bandwidth in the course of quality inspections of ICS repeater systems.
\end{abstract}

KEYWORDS

Bayesian approach, regression, conjugate priors, ICS repeater, Markov chain Monte Carlo.

\section{Introduction}

An interference cancellation system (ICS) telecommunication repeater has been used to detect radio frequency interference during feedback between antennas using a digital signal process. In the case of an ICS repeater with wideband code division multiple access (WCDMA), every telecommunication company uses a different bandwidth of its own conversion [1]. In domestic applications in Korea, specific frequency bandwidths are assigned to each of telecommunication companies for the ICS repeater. Quality engineers inspect only a specific bandwidth assigned to each of telecommunication companies. However, in foreign applications, the frequency bandwidths assigned to a telecommunication company are not unique, depending on the regions in which the ICS repeaters are used. For example, AT\&T agency is assigned at frequency bandwidth 1 in region A of US, while assigned at frequency bandwidth 2 in region B as shown in Fig. 1.

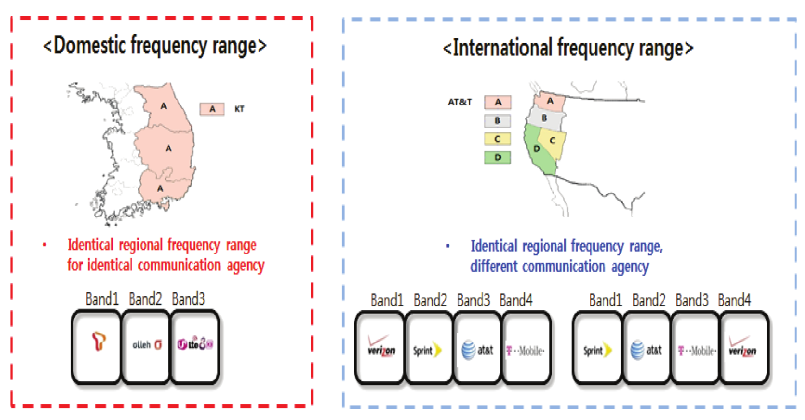

Fig. 1. Domestic and international allocations of frequency bandwidths for ICS repeaters. 
In the case of a WCDMA switched ICS repeater, each of the bandwidths should be inspected manually and individually by an inspector. Hence the inspection procedure is a time consuming procedure resulting in decreasing productivity. Inspection schemes of this repeater system for international applications need a lot of inspection time because quality engineers should inspect all frequency signals at different bandwidths using a measuring instrument. This results in higher inspection costs because all of the bandwidths should be inspected manually [2].

In this study, we introduce a Bayesian regression model to improve productivity by reducing inspection costs caused by repeated measurements. We measure one bandwidth for an ICS repeater, then predict the rest of the adjoining bandwidths using a correlation structure between the measurements via a Bayesian regression model, which reduces measurement times. In general, we select small sample sizes out of a population in quality inspection, and make decisions based on the inspection results from the sample in real applications of ICS repeaters. When prior information is available, Bayesian approaches have provided better estimation results for the parameters of regression models than popular maximum likelihood (ML) methods, especially when the sample size is small [3-5]. For the band selective repeater systems, we expect to increase prediction accuracy for the measurements of adjoining bandwidths of small-sized samples via the proposed Bayesian virtual metrology.

In the literature review, Chen et al. [6] proposed a measurement prediction of the variables in semiconductor manufacturing processes by virtual metrology to improve process control. Khan et al. [7] applied a virtual metrology approach to wafer-towafer monitoring at factory level. There have been a number of other applications of Bayesian approaches throughout various industries. The application of Bayesian methods include estimation of storage reliability on pyrotechnic mechanical devices [8], underwater wireless sensor networks analysis with portstarboard ambiguity [9], single-cell differential expression analysis[10], and subway vibration power analysis on electromotive force [11]. However, to our knowledge, the application of a Bayesian method to the virtual metrology area has not been tried yet.

\section{Bayesian regression model}

As one of the oldest methods in mathematical statistics, a regression analysis is the approach for modeling the relationship between a dependent variable $(y)$ and one or more independent variables $\left(x^{\prime} s\right)$.
Given a data set with $p$ independent variables of $n$ units, a linear regression model assumes the relationship between the $y_{i}$ and $x_{i}{ }^{\prime} s$ as

$$
y_{i}=\beta_{0}+\beta_{1} x_{i 1}+\ldots+\beta_{p} x_{i p}+\varepsilon_{i}, \quad i=1, \ldots, n .
$$

Define $\boldsymbol{x}_{i}=\left(1, x_{i 1}, \ldots, x_{i p}\right)^{T}, \boldsymbol{\beta}=\left(\beta_{0}, \beta_{0}, \ldots, \beta_{p}\right)^{T}$, then a linear regression model (1) can be written as

$$
y_{i}=\boldsymbol{x}_{i}^{T} \boldsymbol{\beta}+\epsilon_{i}, \quad i=1, \ldots, n .
$$

In this formula, we can write the model (2) as a matrix form as

$$
\boldsymbol{y}=\boldsymbol{X} \boldsymbol{\beta}+\boldsymbol{\varepsilon},
$$

where $\boldsymbol{y}=\left(y_{1}, \ldots, y_{n}\right)^{T}, \boldsymbol{X}=\left(\boldsymbol{x}_{1}, \ldots, \boldsymbol{x}_{n}\right)^{T}$, and $\varepsilon=\left(\epsilon_{1}, \ldots, \epsilon_{n}\right)^{T}$. Under normality assumptions for the error terms, ordinary least squares (OLS) estimates of regression coefficients, $\widehat{\boldsymbol{\beta}}_{\mathrm{OLS}}=$ $\left(\boldsymbol{X}^{T} \boldsymbol{X}\right)^{-1} \boldsymbol{X}^{T} \boldsymbol{y}$, are the same as maximum likelihood estimates (MLEs).

If prior information is available, Bayesian estimates for the regression parameters have proven to be better than MLEs, especially for small-sized samples [12]. In this study, we construct a regression model in a Bayesian framework to increase estimation accuracy of regression parameters, and then compared the estimation results with those from OLS method. Prior distributions for the regression parameters are assumed to follow conjugate prior distributions as

$$
\boldsymbol{\beta} \sim M N\left(\boldsymbol{\beta}_{0}, \boldsymbol{\Sigma}_{0}\right), \quad \sigma^{2} \sim I G(a, b),
$$

where $\boldsymbol{\beta}_{0}, \boldsymbol{\Sigma}_{0}$ are the mean and variance of a multivariate normal prior for regression coefficients $\beta$, and $a, b$ are the shape and scale parameters respectively, for an inverse-gamma prior distribution. The multivariate normal probability density function (pdf) is given by

$$
\begin{gathered}
\mathrm{p}\left(\boldsymbol{\beta} \mid \boldsymbol{\beta}_{0}, \boldsymbol{\Sigma}_{0}\right)=(2 \pi)^{-\mathrm{p} / 2}\left|\boldsymbol{\Sigma}_{0}\right|^{-1 / 2} \exp \\
\left\{-\frac{1}{2}\left(\boldsymbol{\beta}-\boldsymbol{\beta}_{0}\right)^{\mathrm{T}} \boldsymbol{\Sigma}_{0}^{-1}\left(\boldsymbol{\beta}-\boldsymbol{\beta}_{0}\right)\right\}, \quad \mathbf{x} \in \mathbb{R}^{\mathrm{p}} .
\end{gathered}
$$

We need a family of prior distributions for $\sigma^{2}$ that has the support $(0, \infty)$. One of such family distributions is the gamma family, hence we assume the prior distribution for $\sigma^{2}$ to be an inverse-gamma distribution; that is, $1 / \sigma^{2} \sim \operatorname{Gamma}(a, b)$. The inversegamma pdf is given by

$$
\mathrm{p}\left(\sigma^{2} \mid \mathrm{a}, \mathrm{b}\right)=\frac{b^{a}}{\Gamma(a)} \sigma^{2^{-(a+1)}} e^{-b / \sigma^{2}}, \quad x \in \mathbb{R},
$$

and the shape and scale parameters for the inversegamma prior distribution can be derived from prior information as

$$
a=v_{0} / 2, \quad b=\left(\sigma_{0}^{2} v_{0}\right) / 2,
$$


where $v_{0}$ and $\sigma_{0}^{2}$ are the degree of freedom for $\sigma^{2}$ and sample variance of prior observations. In general, the degree of freedom for $\sigma^{2}$ is $n-2$ for the simple linear regression and the sample variance $\sigma_{0}^{2}=\mathrm{MSE}=\frac{\mathrm{SSE}}{n-2}$, where SSE is the sum of squares of errors.

The posterior distribution created by combining prior distribution for $\beta$ and $\sigma^{2}$ can be shown as

$$
\begin{aligned}
& p\left(\boldsymbol{\beta}, \sigma^{2} \mid \boldsymbol{y}, \boldsymbol{X}\right) \propto p\left(\boldsymbol{y} \mid \boldsymbol{X}, \boldsymbol{\beta}, \sigma^{2}\right) p\left(\boldsymbol{\beta} \mid \sigma^{2}\right) p\left(\sigma^{2}\right) \\
& \propto\left(\sigma^{2}\right)^{-\frac{n}{2}} \exp \left\{-\frac{1}{2 \sigma^{2}}(\boldsymbol{y}-\boldsymbol{X} \boldsymbol{\beta})^{T}(\boldsymbol{y}-\boldsymbol{X} \boldsymbol{\beta})\right\} \\
& \times\left(\sigma^{2}\right)^{-\frac{k}{2}} \exp \left\{-\frac{1}{2 \sigma^{2}}\left(\boldsymbol{\beta}-\boldsymbol{\beta}_{0}\right)^{\mathrm{T}} \boldsymbol{\Sigma}_{0}\left(\boldsymbol{\beta}-\boldsymbol{\beta}_{0}\right)\right\} \\
& \times\left(\sigma^{2}\right)^{-\left(v_{0} / 2+1\right)} \exp \left\{-\frac{v_{0} \sigma_{0}^{2}}{2 \sigma^{2}}\right\} .
\end{aligned}
$$

We can rewrite Eq. (8) as

$$
\begin{gathered}
\operatorname{p~}\left(\boldsymbol{\beta}, \sigma^{2} \mid \boldsymbol{y}, \boldsymbol{X}\right) \\
\propto\left(\sigma^{2}\right)^{-\frac{k}{2}} \exp \left\{-\frac{1}{2 \sigma^{2}}(\boldsymbol{\beta}-\widetilde{\boldsymbol{\beta}})^{\mathrm{T}}\left(\boldsymbol{X}^{T} \boldsymbol{X}+\boldsymbol{\Sigma}_{0}\right)(\boldsymbol{\beta}-\widetilde{\boldsymbol{\beta}})\right\} \\
\times\left(\sigma^{2}\right)^{-\left(\left(n+v_{0}\right) / 2+1\right)} \exp \left\{-\frac{\left(v_{0} s_{0}^{2}+n s^{2}\right)}{2 \sigma^{2}}\right\}
\end{gathered}
$$

where $\widetilde{\boldsymbol{\beta}}=\left(\boldsymbol{X}^{T} \boldsymbol{X}+\boldsymbol{\Sigma}_{0}\right)^{-1}\left(\boldsymbol{X}^{T} \boldsymbol{X} \widehat{\boldsymbol{\beta}}+\boldsymbol{\Sigma}_{0} \boldsymbol{\beta}_{0}\right)$, and $\boldsymbol{\Sigma}_{\boldsymbol{\beta}}=\sigma_{0}^{2}\left(\boldsymbol{X}^{T} \boldsymbol{X}+\boldsymbol{\Sigma}_{0}\right)^{-1}$. Then the posterior distribution for the parameters are also multivariate normal distribution and inverse-gamma distribution as

$$
\begin{gathered}
\boldsymbol{\beta} \mid \sigma^{2} \boldsymbol{y} \sim M N\left(\widetilde{\boldsymbol{\beta}}, \boldsymbol{\Sigma}_{\boldsymbol{\beta}}\right), \\
\text { and } \quad \sigma^{2} \sim I G\left(v_{0}+n, \frac{v_{0} s_{0}^{2}+n s^{2}}{v_{0}+n}\right),
\end{gathered}
$$

where

$$
n s^{2}=(\boldsymbol{y}-\boldsymbol{X} \widetilde{\boldsymbol{\beta}})^{T}(\boldsymbol{y}-\boldsymbol{X} \widetilde{\boldsymbol{\beta}})+\left(\widetilde{\boldsymbol{\beta}}-\boldsymbol{\beta}_{0}\right)^{T} \boldsymbol{\Sigma}_{0}\left(\widetilde{\boldsymbol{\beta}}-\boldsymbol{\beta}_{0}\right) .
$$

Note that the Bayes estimator corresponding to the posterior mean is $\widetilde{\boldsymbol{\beta}}$.

As another approach, we introduce a multivariate regression model [13-15]. The two neighboring bandwidths can be predicted by measuring an intermediate bandwidth. In a multivariate linear regression for $\boldsymbol{y}$ against $x^{\prime} s$, a set of regression equations related through common $\boldsymbol{x}$ variables and correlated errors is represented as

$$
\boldsymbol{y}_{i}=\boldsymbol{x}_{i}^{T} \boldsymbol{\beta}_{\boldsymbol{i}}+\boldsymbol{\epsilon}_{\boldsymbol{i}}, \quad \boldsymbol{\epsilon}_{\boldsymbol{i}} \sim M N(\mathbf{0}, \boldsymbol{\Sigma}),
$$

where $\boldsymbol{y}_{i}=\left(y_{1 i}, \ldots, y_{m i}\right)^{T}$ is a $(m \times 1)$ response vector for $i$-th observed data, $\boldsymbol{x}_{i}=\left(1, x_{i 1}, \ldots, x_{i p}\right)^{T}$ and $\boldsymbol{\beta}_{\boldsymbol{i}}=\left(\beta_{0 i}, \beta_{1 i}, \ldots, \beta_{p i}\right)^{T}$. The model (11) is a convenient way of expressing the model for the purpose of writing down the likelihood function for the model as

$$
\begin{gathered}
p\left(\boldsymbol{\epsilon}_{\mathbf{1}}, \ldots, \boldsymbol{\epsilon}_{\boldsymbol{n}} \mid \boldsymbol{\Sigma}\right) \propto|\boldsymbol{\Sigma}|^{-\frac{n}{2}} \exp \left\{-\frac{1}{2} \operatorname{tr}\left(\boldsymbol{S}_{\boldsymbol{\epsilon}}\right) \boldsymbol{\Sigma}^{-\mathbf{1}}\right\}, \\
\boldsymbol{S}_{\boldsymbol{\epsilon}}=\sum_{i=1}^{n} \boldsymbol{\epsilon}_{\boldsymbol{i}} \boldsymbol{\epsilon}_{\boldsymbol{i}}^{\boldsymbol{T}} .
\end{gathered}
$$

where $\operatorname{tr}(\cdot)$ denotes a trace of a vector. To obtain the form for the natural conjugate prior for the regression coefficients, we can rewrite the multivariate regression model as,

$$
\boldsymbol{Y}=\boldsymbol{X} \boldsymbol{B}+\boldsymbol{E}, \quad \boldsymbol{B}=\left[\boldsymbol{\beta}_{1}, \ldots, \boldsymbol{\beta}_{\boldsymbol{c}}, \ldots, \boldsymbol{\beta}_{\boldsymbol{m}}\right] .
$$

Both $Y$ and $E$ are $(n \times m)$ matrices of observations whose $(i, j)$-th elements are the $i$-th observation on equation $j . \boldsymbol{X}$ is an $(n \times p)$ matrix of observations on the $p$ common independent variables. To construct the form of the natural conjugate prior, the likelihood function is written as

$$
p(\boldsymbol{Y} \mid \boldsymbol{X}, \boldsymbol{B}, \boldsymbol{\Sigma}) \propto|\boldsymbol{\Sigma}|^{-n / 2}
$$

$\operatorname{etr}\left\{-\frac{1}{2}\left(\boldsymbol{S}+(\boldsymbol{B}-\widehat{\boldsymbol{B}})^{T} \boldsymbol{X}^{\prime} \boldsymbol{X}(\boldsymbol{B}-\widehat{\boldsymbol{B}})\right)^{T} \boldsymbol{\Sigma}^{-1}\right\}$

with $\boldsymbol{S}=(\boldsymbol{Y}-\boldsymbol{X} \widehat{\boldsymbol{B}})^{T}(\boldsymbol{Y}-\boldsymbol{X} \widehat{\boldsymbol{B}}), \widehat{\boldsymbol{B}}$ is the OLS of $\boldsymbol{B} ; \widehat{\boldsymbol{B}}=\left(\boldsymbol{X}^{T} \boldsymbol{X}\right)^{-1} \boldsymbol{X}^{T} \boldsymbol{Y}$. Here, $\operatorname{etr}(\cdot)=\exp (\operatorname{tr}(\cdot))$, where $\operatorname{tr}(\cdot)$ is a trace of a vector. The OLS of Eq. (14) suggests that the natural conjugate prior is an inverted Wishart on $\boldsymbol{\Sigma}$ and a prior on $\boldsymbol{B}$ which is conditional on $\boldsymbol{\Sigma}$. The natural conjugate priors for the multivariate regression model are of the form

$$
\begin{gathered}
p(\boldsymbol{\Sigma}, \boldsymbol{B})=p(\boldsymbol{\Sigma}) p(\boldsymbol{B} \mid \boldsymbol{\Sigma}), \quad \boldsymbol{\Sigma} \sim I W\left(\boldsymbol{v}_{0}, \boldsymbol{V}_{0}\right), \\
\boldsymbol{B} \mid \boldsymbol{\Sigma} \sim N\left(\widetilde{\boldsymbol{\beta}}, \boldsymbol{\Sigma} \otimes \boldsymbol{\Sigma}_{\boldsymbol{\beta}}^{-1}\right),
\end{gathered}
$$

where $\otimes$ is an operation on two matrices of arbitrary size resulting in a block matrix; that is a Kronecker product. Just as in the univariate regression model, the prior on the regression coefficients is dependent on the scale parameters. The posterior can be obtained by combining terms from the natural conjugate prior to produce a posterior which is a product of an inverted Wishart and a 'matrix' normal kernel as

$$
\begin{gathered}
p(\boldsymbol{\Sigma}, \boldsymbol{B} \mid \boldsymbol{Y}, \boldsymbol{X}) \propto|\boldsymbol{\Sigma}| \operatorname{etr}\left(-\frac{1}{2} \boldsymbol{V}_{0} \boldsymbol{\Sigma}^{-1}\right) \\
\times|\boldsymbol{\Sigma}|^{-\frac{k}{2}} \operatorname{etr}\left(-\frac{1}{2}(\boldsymbol{B}-\overline{\boldsymbol{B}})^{T} \boldsymbol{\Sigma}_{0}(\boldsymbol{B}-\overline{\boldsymbol{B}}) \boldsymbol{\Sigma}^{-1}\right) \\
\times|\boldsymbol{\Sigma}|^{-k / 2} \operatorname{etr}\left(-\frac{1}{2}(\boldsymbol{Y}-\boldsymbol{X} \boldsymbol{B})^{T}(\boldsymbol{Y}-\boldsymbol{X} \boldsymbol{B}) \boldsymbol{\Sigma}^{-1}\right) .
\end{gathered}
$$

We can combine the two terms involving $\boldsymbol{B}$, using the same structure for the univariate regression model as 


$$
\begin{aligned}
(\boldsymbol{B}-\overline{\boldsymbol{B}})^{T} & \boldsymbol{\Sigma}_{0}(\boldsymbol{B}-\overline{\boldsymbol{B}})+(\boldsymbol{Y}-\boldsymbol{X} \boldsymbol{B})^{T}(\boldsymbol{Y}-\boldsymbol{X} \boldsymbol{B}) \\
& =(\boldsymbol{Z}-\boldsymbol{W} \boldsymbol{B})^{T}(\boldsymbol{Z}-\boldsymbol{W} \boldsymbol{B}) \\
& =(\boldsymbol{Z}-\boldsymbol{W} \widetilde{\boldsymbol{B}})^{T}(\boldsymbol{Z}-\boldsymbol{W} \widetilde{\boldsymbol{B}}) \\
+ & (\boldsymbol{B}-\widetilde{\boldsymbol{B}})^{T} \boldsymbol{W}^{\prime} \boldsymbol{W}(\boldsymbol{B}-\widetilde{\boldsymbol{B}}) .
\end{aligned}
$$

With $\boldsymbol{W}=\left[\begin{array}{c}\boldsymbol{X} \\ \boldsymbol{U}\end{array}\right], \boldsymbol{Z}=\left[\begin{array}{l}\boldsymbol{Y} \\ \boldsymbol{U} \overline{\boldsymbol{B}}\end{array}\right], \boldsymbol{\Sigma}_{0}=\boldsymbol{U}^{T} \boldsymbol{U}$.

The posterior density can now be written as

$$
\begin{gathered}
p(\boldsymbol{\Sigma}, \boldsymbol{B} \mid \boldsymbol{Y}, \boldsymbol{X}) \propto \\
|\boldsymbol{\Sigma}|^{-\left(v_{0}+n+m+1\right) / 2} \\
\operatorname{etr}\left(-\frac{1}{2}\left(\boldsymbol{V}_{0}+(\boldsymbol{V}-\boldsymbol{W} \widetilde{\boldsymbol{B}})^{T} \boldsymbol{\Sigma}^{-1}\right)\right) \\
\times|\boldsymbol{\Sigma}|^{-k / 2} \operatorname{etr}\left(-\frac{1}{2}(\boldsymbol{B}-\widetilde{\boldsymbol{B}})^{T} \boldsymbol{W}^{\prime} \boldsymbol{W}(\boldsymbol{B}-\widetilde{\boldsymbol{B}})\right) .
\end{gathered}
$$

Thus, the posterior is in the form of the conjugate prior as

$$
\begin{gathered}
\boldsymbol{\Sigma} \mid \boldsymbol{Y}, \boldsymbol{X} \sim I W\left(v_{0}+n, \boldsymbol{V}_{0}+\boldsymbol{S}\right) \\
\boldsymbol{\beta} \mid \boldsymbol{Y}, \boldsymbol{X}, \boldsymbol{\Sigma} \sim N\left(\widetilde{\boldsymbol{\beta}}, \boldsymbol{\Sigma} \otimes\left(\boldsymbol{X}^{T} \boldsymbol{X}+\boldsymbol{A}\right)^{-1}\right) \\
\widetilde{\boldsymbol{\beta}}=\operatorname{vec}(\widetilde{\boldsymbol{B}}) \\
\widetilde{\boldsymbol{B}}=\left(\boldsymbol{X}^{T} \boldsymbol{X}+\boldsymbol{A}\right)^{-1}\left(\boldsymbol{X}^{T} \boldsymbol{X} \widehat{\boldsymbol{B}}+\boldsymbol{A} \overline{\boldsymbol{B}}\right) \\
\boldsymbol{S}=(\boldsymbol{Y}-\boldsymbol{X} \widetilde{\boldsymbol{B}})^{T}(\boldsymbol{Y}-\boldsymbol{X} \widetilde{\boldsymbol{B}})+(\widetilde{\boldsymbol{B}}-\overline{\boldsymbol{B}})^{T} \boldsymbol{A}(\widetilde{\boldsymbol{B}}-\overline{\boldsymbol{B}}) .
\end{gathered}
$$

\section{Measuring methods and data set}

ICS repeaters are inspected using spectrum analysis tools after calibration of a measuring instrument. The instrument inspects the shape and dimension of the frequency produced in the ICS repeater. The ideal frequency shape is of rectangular form, and the normal frequency shape is determined by the height from the bottom of the frequency as shown in Fig. 2. The ICS repeater will fail if measured values of frequency height lie outside of the margin limits.

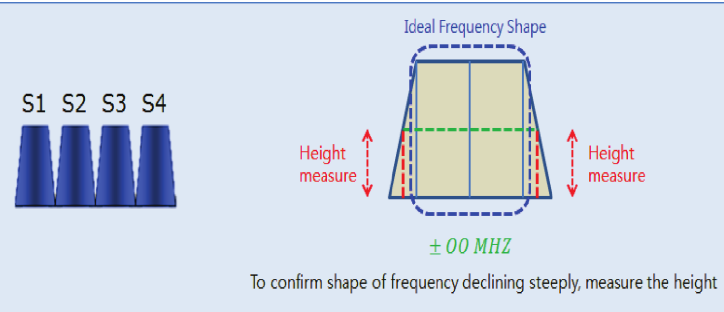

Fig. 2. Measuring scheme for the frequency bandwidth for an ICS repeater system.
The data set in our research consisted of a total of 210 measurement data for ICS repeater systems. Each data contains three variables measured at three different bandwidths. Each variable represents measured frequency height on a frequency bandwidth of an ICS repeater. Because the data were measured at different frequency bandwidths repeatedly with respect to the same components (heights), measurement time increased as frequency bands that should be inspected increased. Figure 3 shows the clear correlation structure among the three bands for 210 repeater systems.

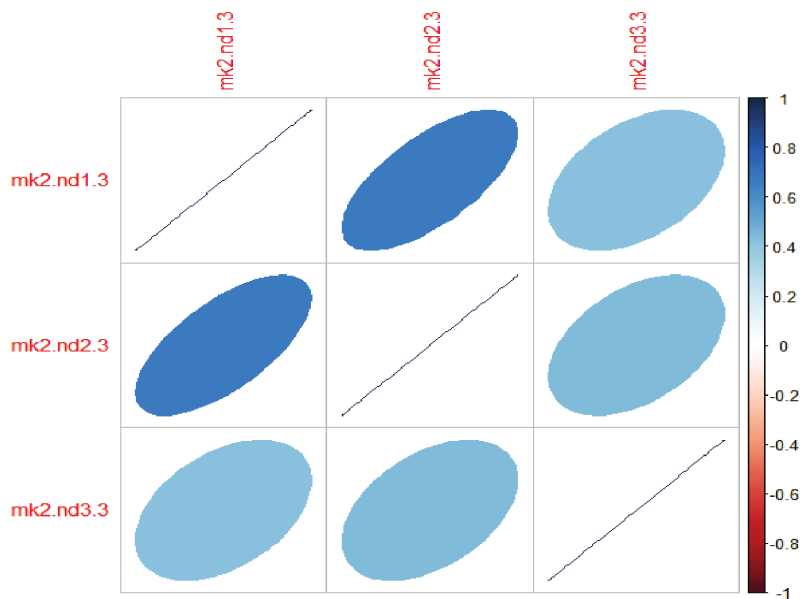

Fig. 3. Correlation plot.

Table 1 describes the data set in our analysis. Contrary to general linear regression approaches, Bayesian estimation draws a posterior distribution of the parameters from prior distributions.

Table 1

Data set structure for experiment.

\begin{tabular}{c|c}
\hline The purpose of data & The number of data \\
\hline Liner regression $(n=50)$ & $y=50, x=50$ \\
\hline Liner regression $(n=30)$ & $y=30, x=30$ \\
\hline Liner regression $(n=5)$ & $y=5, x=5$ \\
\hline Data for producing prior information & $y=100, x=100$ \\
\hline
\end{tabular}

We considered three cases: $n=5,30$, and 50. For all of the cases, the prior distributions for the regression parameters are constructed by historical data with the sample size 100 .

\section{Analytical results}

We compared Bayesian estimates of the regression parameters with ordinary least square (OLS) estimates in a simple linear regression. We conducted random sampling to select 30 samples from 100 data set and set up parameters $\boldsymbol{\beta}_{0}$ and $\boldsymbol{\Sigma}_{0}$ for the multivariate normal prior distribution. We repeated this 50 times with the sample size of 30 . Then, we 
conducted a regression analysis with each of the 50 sample sets and calculated the mean and variance for regression coefficients $\boldsymbol{\beta}$, then set up the results for parameters for prior distributions $\boldsymbol{\beta}_{0}$ and $\boldsymbol{\Sigma}_{0}$. We set $v_{0}=28$ and $\left(\sigma_{0}^{2} v_{0}\right) / 2=\frac{\mathrm{MSE} * v_{0}}{2}=50$ for the sample set. From these posterior distributions, we estimated parameters and compared them to parameters that were created by a linear regression with $n=50, n=30$, and $n=5$.

As can be seen in Table 2, standard errors for regression coefficients increase as the data size decreases in OLS estimates. However, in the case of a Bayesian regression, the estimation errors were kept very small by incorporating prior information in estimating the parameters, even $n=5$. Figure 4 shows the distribution for the regression coefficients in Bayesian linear regression model. The coefficients $\beta_{0}$ and $\beta_{1}$ in linear regression models are plotted when sample sizes are 5, 30 and 50. Comparing the modeling accuracy between ordinary linear regression and Bayesian linear regression when the sample is small ( $n=5)$, the Bayesian regression with prior information estimates more accurately, indicating lower Std. Error (0.335) than the one with LSE (10.810), even on small-sized sample conditions. We also could identify that posterior distribution is more likely to be influenced by prior information. Accord-
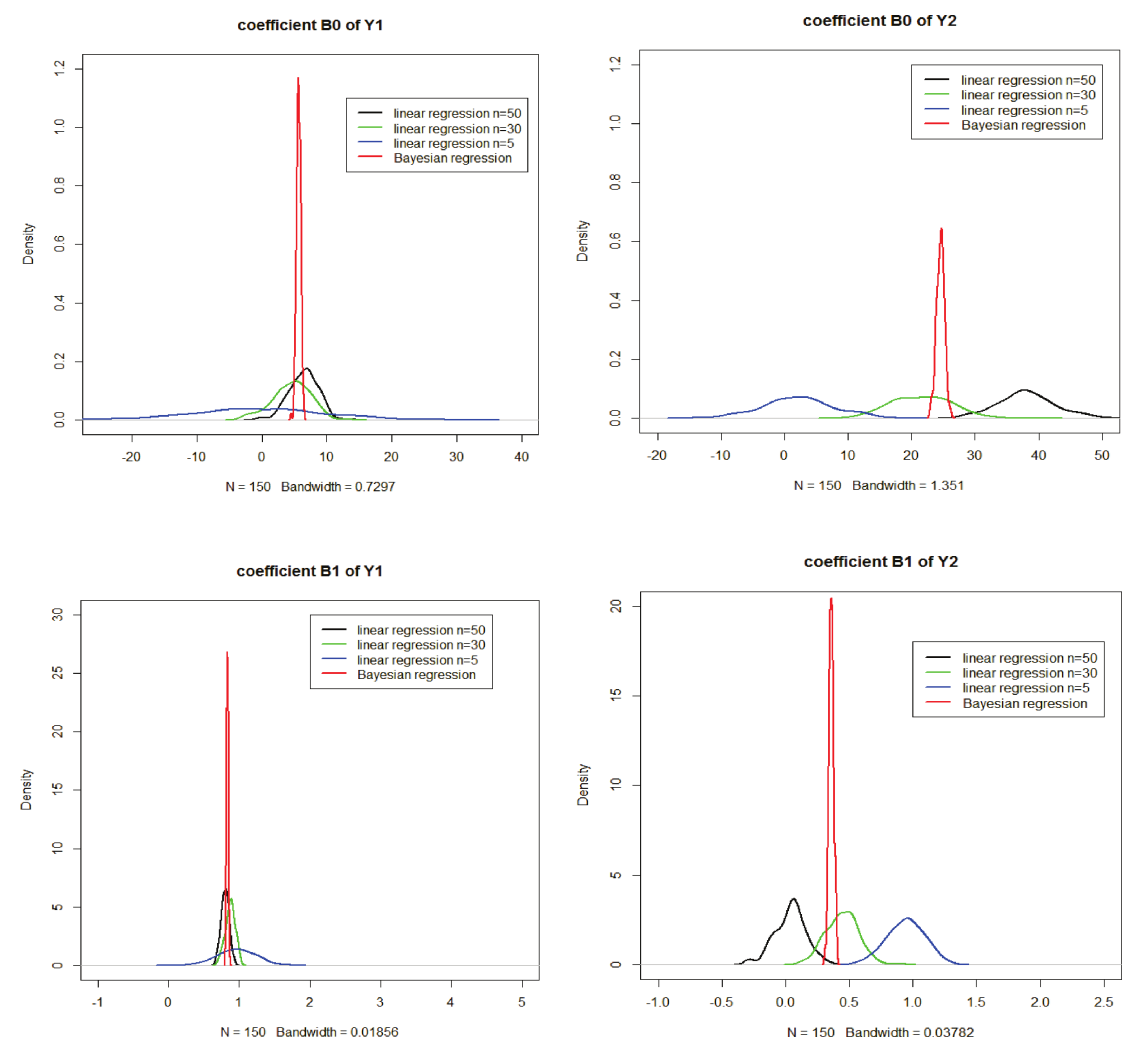

Fig. 4. The distribution comparisons for regression coefficients in Bayesian multivariate regression.

ingly, once accurate prior information is acquired, more precise estimations on regression coefficients would be possible by applying the Bayesian method even on small-sized sample conditions.

Next, we compared the parameter estimation results from OLS method with those from Bayesian multivariate regression model. Table 2 shows that even if the sample size is as small as five, the esti-

Table 2

Bayesian multivariate regression result.

\begin{tabular}{c|c|c|c}
\hline Parameter & Method & Mean & Std. Error \\
\hline \multirow{3}{*}{$\boldsymbol{\beta}_{0}(\boldsymbol{Y 1})$} & Linear regression $(n=30)$ & 4.6285 & 3.1361 \\
\cline { 2 - 4 } & Linear regression $(n=50)$ & 6.7215 & 2.4165 \\
\cline { 2 - 4 } & Linear regression $(n=5)$ & 0.2560 & 10.8097 \\
\cline { 2 - 4 } & Bayesian regression & $\mathbf{5 . 7 1 4 3}$ & $\mathbf{0 . 3 8 0 9}$ \\
\hline \multirow{3}{*}{$\boldsymbol{\beta}_{0}(\boldsymbol{Y 2})$} & Linear regression $(n=50)$ & 38.3915 & 4.9099 \\
\cline { 2 - 4 } & Linear regression $(n=30)$ & 21.2252 & 5.0394 \\
\cline { 2 - 4 } & Linear regression $(n=5)$ & 3.0636 & 5.4096 \\
\cline { 2 - 4 } & Bayesian regression & $\mathbf{2 4 . 5 6 6 7}$ & $\mathbf{0 . 5 1 4 5}$ \\
\hline \multirow{3}{*}{$\boldsymbol{\beta}_{1}(\boldsymbol{Y 1})$} & Linear regression $(n=50)$ & 0.8066 & 0.0626 \\
\cline { 2 - 4 } & Linear regression $(n=30)$ & 0.8624 & 0.0821 \\
\cline { 2 - 4 } & Linear regression $(n=5)$ & 0.9813 & 0.2822 \\
\cline { 2 - 4 } & Bayesian regression & $\mathbf{0 . 3 5 9 8}$ & $\mathbf{0 . 0 1 3 2}$ \\
\hline \multirow{3}{*}{$\boldsymbol{\beta}_{1}(\boldsymbol{Y 2})$} & Linear regression $(n=50)$ & 0.0133 & 0.1272 \\
\cline { 2 - 4 } & Linear regression $(n=30)$ & 0.4504 & 0.1320 \\
\cline { 2 - 4 } & Linear regression $(n=5)$ & 0.9252 & 0.1412 \\
\cline { 2 - 4 } & Bayesian regression & $\mathbf{0 . 3 5 9 7}$ & $\mathbf{0 . 0 1 7 3}$ \\
\hline
\end{tabular}

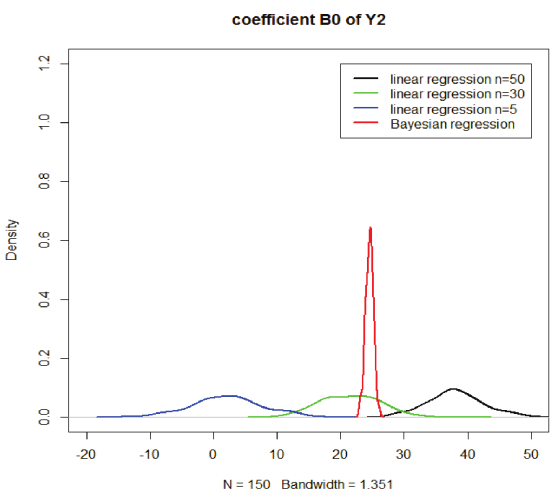


mation accuracy of regression coefficients increases when we incorporate prior information in estimating regression parameters. Figure 4 also shows that the Bayesian multivariate regression model provides more reliable regression results.

Overall, in comparison with the results from OLS method, the Bayesian multivariate linear regression shows higher accuracy on estimation of regression coefficients than in Bayesian simple linear regressions.

\section{Conclusion}

A virtual metrology approach in our applications enables manufacturers to significantly reduce measuring times and costs in quality inspections of ICS repeater systems. Testing methods of mobile repeater systems require repeated measurements for different frequency band sections. The current inspection scheme results in lower productivity by requiring a repeated application of the same measuring methods to other different frequency band sections. This paper proposes an application of Bayesian regression methods based on correlated measurement data and predictions of the other frequency band sections without direct measurements. We confirmed that higher estimation accuracy is possible when such a Bayesian regression model is applied, even with a with small-sample size. Measuring times and costs can be reduced by properly setting prior information for the regression parameters.

In future research, a beneficial circle would be created through an economy of scale that raises the volume of sales while lowering production costs and product prices. Additionally, further research is needed on other measurement items of ICS repeaters beyond the ones currently investigated. Other methodologies should also be explored for measurement items that have upper or lower limits, not only target values.

We thank the referees who helped us gain substantial improvements in the manuscript. This work was supported by Basic Science Research Program through the National Research Foundation of Korea funded by the Ministry of Education (No. 2015R1D1A1A01059799). This work was supported by the Human Resources Program in Energy Technology of the Korea Institute of Energy Technology Evaluation and Planning (KETEP), granted financial resource from the Ministry of Trade, Industry 86 Energy, Republic of Korea (No. 20154030200900).

\section{References}

[1] Hoff P.D., A first course in Bayesian statistical methods, Springer, 2009.
[2] Perez-Elizalde S., Cuevas J., Cross J., Selection of the bandwidth parameter in a Bayesian kernel regression model for genomic-enabled prediction, Journal of Agricultural, Biological, and Environmental Statistics, 20, 4, 512-532, 2015.

[3] Biller C., Adaptive Bayesian regression splines in semiparametric generalized linear models, Journal of Computational and Graphical Statistics, 9, 1, 122 $140,2000$.

[4] Zougab N., Adjabi S., Kokonendji C., A Bayesian approach to bandwidth selection in univariate associate kernel estimation, Journal of Statistical Theory and Practice, 7, 1, 8-23, 2013.

[5] Rossi P.E., Bayesian statistics and marketing, Jonh Wiley \& Sons, 2005.

[6] Chen P.H., Wu S., Lin J.S., Ko F., Lo H., Wang J., Yu C.H., Liang M.S., Virtual metrology: a solution for wafer to wafer advanced process control, IEEE International Symposium on Semiconductor Manufacturing, 155-157, 2005.

[7] Khan A.A., Moyne J.R., Tilbury D.M., An approach for factory-wide control utilizing virtual metrology, IEEE Transactions on Semiconductor Manufacturing, 20, 4, 364-375, 2007.

[8] Mun B.M., Lee C.U., Kim J.S., Ku J.R., Kim Z.I., Bae S.J., A Bayesian approach to predict storage reliability for pyrotechnic mechanical device, KSPE Spring Conference, 2015.

[9] Braca P., Willett P., LePage K., Marano S., Matta V., Bayesian tracking in underwater wireless sensor networks with port-starboard ambiguity, IEEE Transactions on Signal Processing, 62, 7, 1864-1878, 2014.

[10] Kharchenko P., Silberstein L., Scadden D., Bayesian approach to single-cell differential expression analysis, Nature Methods, 11, 7, 740-744, 2014.

[11] Jo B.W., Kim Y.S., Kim Y.G., A fundamental study on analysis of electromotive force and updating of vibration power generating model on subway through the Bayesian regression and correlation analysis, Computational Structural Engineering Institute of Korea, 26, 2, 139-146, 2013.

[12] Berger J., Statistical Decision Theory and Bayesian Inference, Springer-Verlag, Berlin, 1985.

[13] Arroyo D., Ordaz M., Multivariate Bayesian regression analysis applied to pseudo-acceleration attenuation relationships, The 14 th World Conference on Earthquake Engineering, 2008.

[14] Rowe D.B., Multivariate Bayesian statistics models for source separation and signal unmixing, Chapman \& Hall/CRC, 2003.

[15] Tiao G.C., Zellner A., On the Bayesian estimation of multivariate regression, Journal of the Royal Statistical Society, 26, 2, 277-285, 1964. 